\title{
Representações sociais e o papel terapêutico dos acadêmicos de enfermagem
}

\author{
Social representations and the therapeutic role of nursing students
}

Las representaciones sociales y el rol terapéutico de estudiantes de enfermería

\author{
Francisco Arnoldo Nunes de Miranda', Clélia Albino Simpson', Rafaella Leite Fernandes', \\ Marta Batista Silva", Maria das Graças Garcia Sabino"II \\ 'Universidade Federal do Rio Grande do Norte. Programa de Pós-Graduação em Enfermagem. Natal, RN \\ "Ministério da Saúde. Brasília, DF \\ II'Secretaria de Estado da Saúde Pública. Centro de Apoio Psicossocial. Natal, RN
}

Submissão: 04/05/2009

Aprovação: 3 1/08/2009

\section{RESUMO}

O presente estudo de abordagem Qualitativa objetiva apreender as representações sociais e práticas disciplinares em Saúde Mental do papel terapêutico do acadêmico de enfermagem na atenção ao adulto no Contexto da Clínica Ampliada realizadas no Hospital-Dia Dr. Elger Nunes, Natal/RN. Analisamos os 42 estudos de caso, formatados em um corpus a luz do programa informático ALCESTE. Inferimos Que para este grupo de alunos a representação social é estabelecida entre adQuirir conhecimento para ser terapêutico como aluno e profissional. Esta competência o torna hábil para lidar com os transtornos mentais na perspectiva deste serviço, assim como no modelo oficial de atenção à saúde.

Descritores: Hospital dia; Estudantes de enfermagem; Saúde mental; Transtornos mentais; Enfermagem.

\section{ABSTRACT}

This study with a Qualitative approach aims to apprehend the social representation and disciplinary practices in Mental Health subject regarding the therapeutic role of the nursing students in the attention of adult into the Context of Enlarged Clinic held in the Day-Hospital Dr. Elger Nunes, Natal / RN. A total of 42 case studies were analyzed and formatted in corpus and based on the ALCESTE software. It was inferred for this group of students that the social representation is established aceuiring knowledge to be therapeutic as student and professional. This competence makes them skilled to deal with mental disorders from the perspective of this service, as well as the official model of health care.

Descriptors: Hospitals, day; Students, nursing; Mental health; Mental disorders; Nursing.

\section{RESUMEN}

Este estudio con abordaje cualitativo se propone identificar las representaciones sociales y las prácticas disciplinares en Salud Mental del rol terapéutico de los académicos de enfermería en la atención a los adultos en el Contexto de la Clínica Ampliada, realizada en el Hospital Día Dr. Elger Nunes, Natal / RN. Analizamos los 42 estudios de caso, formateados en un corpus bajo la luz de software ALCESTE. Se infiere Que para este grupo de estudiantes las representaciones sociales se establece entre la adQuisición de conocimientos para ser terapéutico como estudiantes y profesionales. Esta competencia capacita los estudiantes a tratar los trastornos mentales desde la perspectiva de esto servicio, así como en el modelo oficial de la asistencia a salud.

Descriptores: Hospitales de Día; Estudiantes del enfermería; Salud Mental; Trastornos mentales.; Enfermería 


\section{INTRODUÇÃO}

As diretrizes curriculares exigem mecanismos e estratégias didático-pedagógicas capazes de garantir ao egresso um exercício profissional na assistência, ensino, pesquisa com vistas ao gerenciamento dos serviços de enfermagem e de saúde centrada nas habilidades e competências. Dessa forma, o Curso de Graduação em Enfermagem da Universidade Federal do Rio Grande do Norte - UFRN se insere.

O aluno é estimulado a se perceber, de um lado, como sujeitoobjeto da aprendizagem, do outro, como sujeito-objeto Que se comunica com contexto das práticas disciplinares em saúde mental e psiquiatria, capaz de exteriorizar sentimentos e aliviar possíveis efeitos ansiogênicos de si e do confronto das intervenções em saúde mental e psiquiatria.

Neste estudo objetivamos apreender as representações sociais através das práticas disciplinares no papel terapêutico do acadêmico de enfermagem sobre Saúde Mental na atenção ao adulto no Contexto da Clínica Ampliada realizadas no Hospital-Dia Dr. Elger Nunes, Natal/RN.

\section{REVISÃO E APROXIMAÇÃO CONCEITUAL}

A Organização Mundial de Saúde chama a atenção dos profissionais de saúde e dos gestores das políticas públicas de saúde para o real ônus dos transtornos mentais e comportamentais para seus custos em termos humanos, sociais e econômicos, além da estigmatização, da discriminação e da insuficiência dos serviços de saúde Que impedem milhões de pessoas em todo o mundo de receber tratamento de Que necessitam e Que merecem ${ }^{(1)}$.

A Reforma PsiQuiátrica através da Lei 10.2 16, de 06.04.200 I determina Que os pacientes há longo tempo hospitalizado sejam (re)inseridos em núcleo familiar (de origem ou substitutas) como forma de reduzir progressivamente os leitos psiQuiátricos, além de Qualificar, expandir e fortalecer a rede extra-hospitalar por meio dos Centros de Atenção Psicossocial (CAPS), Serviços Residenciais Terapêuticos (SRTs) e Unidades PsiQuiátricas em Hospitais Gerais (UPHG) - e incluir as ações de saúde mental na atenção básica e Saúde da Família ${ }^{(2)}$.

A substituição do modelo hospitalocêntrico, centrado na doença, na exclusão social, intramuros, por um novo modelo de atenção descentralizado e de base comunitária, exigirá também uma adaptação dos profissionais, Que deverão, através do processo de capacitação, se instrumentalizarem para permitir a viabilização do novo modelo assistencial ${ }^{(3)}$.

Nesse sentido, as representações sociais são uma forma de conhecimento socialmente elaborado e compartilhado, com um objetivo prático, e Que contribui para a construção de uma realidade comum a um conjunto social ${ }^{(4)}$. É um corpus organizado de conhecimentos, resultante das atividades psíquicas pelas Quais os homens tornam a realidade física e social inteligível, possibilitando sua inscrição num dado grupo através da relação cotidiana de trocas $^{(5)}$.

Tem-se observado a progressiva freqüência do conceito de representação social em diversas áreas ${ }^{(6)}$. Uma das razões para sua proliferação é o fato de não constituir um patrimônio de uma área em particular, reconhecendo suas raízes na sociologia, além da presença marcante na antropologia e na história das mentalidades, assim como na psicologia social.

Toda representação social está organizada em torno de um núcleo central e de elementos periféricos ${ }^{(7,8)}$. A natureza do núcleo central é essencialmente social; liga-se às condições históricas, sociológicas e ideológicas, diretamente associadas aos valores e normas. O sistema periférico associa-se às características individuais e ao contexto imediato e contingente, o Que permite adaptações e diferenciações em função do vivido, integrando as experiências cotidianas às informações e até práticas diferenciadas. Dessa forma, emerge a Teoria do Núcleo Central ${ }^{(8)}$.

\section{METODOLOGIA}

Nas práticas disciplinares em saúde mental, cada aluno registra diariamente num impresso roteirizado as atividades e os cuidados de enfermagem realizados no Hospital-Dia Dr. Elger Nunes. Estes registros subsidiam o Estudo de Caso. Utilizamos os Estudos de Casos desenvolvidos no primeiro semestre letivo de 2004 pelos acadêmicos do Quinto período do curso de Graduação Enfermagem da UFRN.

Os dados transformados em corpus foram submetidos ao programa informático denominado "Analyse Lexicale par Contexte d'un Ensemble de Segments de Texte" (ALCESTE), considerado pioneiro no uso da informática na análise de conteúdo ${ }^{(9)}$.

Em função de algumas características do material coletado, tais como várias entrevistas, respostas e número de participantes em Questões abertas, cada resposta será considerada uma Unidade de Comando Inicial ( $\mathrm{UCl}$ ) ou, "linha de comando", "linha com asteriscos" $^{\prime(10)}$. Consideramos como $\mathrm{UCl}$ cada estudo de caso. $\mathrm{Na}$ seQüência nomeamos o corpus (ATUAÇÃO_TERAPÊUTICA _DE_ENFERMAGEM) e definimos as variáveis como: aluno de enfermagem $=$ aenf, histórico $=$ hist, Problemas detectados $=$ pdec, Prescrição de enfermagem = penf, Justificativa de enfermagem $=$ jenf, Evolução = evol.

O ALCESTE faz a análise léxica das palavras de um conjunto de textos, independente da origem de sua produção, Quer das comunicações, Quer das manifestações discursivas, neste caso os estudos de caso. Agrupa as raízes semânticas definindo-as por classes levando em consideração a função da palavra dentro de um dado texto. Tanto é possível Quantificar, como inferir sobre a delimitação das classes, as Quais são definidas em função da ocorrência e da co-ocorrência das palavras e da sua função textual, até aQui sem nenhuma interferência do pesquisador e ainda dar pistas das representações sociais.

\section{RESULTADOS E DISCUSSÃO}

\section{Interface das Representações Sociais e as Práticas de Enfermagem na Saúde Mental}

As imagens geradas pelo ALCESTE são divididas por dois eixos, um horizontal e o outro perpendicular, ou seja, as abscissas $\underline{x}$ e $y$, formando Quatro partes iguais, doravante considerados Quadrantes. Neles localizam-se as unidades semânticas significantes e distribuídas pelo programa sobre o Que se Quer pesquisar. Os espaços geométricos de cada imagem indicam campos geradores de 
categorias e dão pistas para imersão das representações sociais.

Apresentaremos somente uma das três figuras geradas pelo ALCESTE, por ser aquela Que contém as raízes léxicas formadoras das classes e definidoras na composição dos Quadrantes. Não menos importante, as duas outras dizem respeito à distribuição dos sujeitos pesquisados e as variáveis definidas. Dessa forma, é-nos possível localizar o conjunto de falas de cada sujeito sobre cada aspecto abordado na coleta de dados e transformados em $\mathrm{UCl}$, unidade de contexto inicial, na preparação do corpus.

Os achados gerados pelo ALCESTE (versão4. 5 - 01/10/99) foram analisados a partir dos perfis das classes compostas pelas Unidades de Contexto Elementar, denominada U.C.E. e os respectivos Qui-Quadrados, entendidos como identificação dos sujeitos da pesquisa, anteriormente descrito. Nesse sentido, o texto será destacado em itálico e ao final entre parêntese especificamos o número da UCE e o valor do Qui-Quadrado correspondente.

A distribuição léxica das formas originais por raiz Quadradas é agrupada por classes, configurando o contexto de uma mesma classe, podendo também participar na configuração de outra em função dos cruzamentos estatísticos. Portanto, o primeiro Quadrante, o superior direito (QSD) é formado pelas classes $C>$ A > F; o segundo, o Quadrante superior direito (QSD) pela classe $\mathrm{D}>\mathrm{C}$; o terceiro Quadrante o inferior esquerdo (QIE) pelas classes $\mathrm{F}>\mathrm{D}>\mathrm{A}$; e finalmente, o Quarto Quadrante o inferior direito (QDI) pelas classes B $>$ D $>$ E. Estabelecemos exemplos de falas para cada um dos quadrantes constituídos das classes geradoras do núcleo central, os elementos periféricos e os elementos intermediários (Figura I).

O núcleo central estruturante ou núcleo duro determina a natureza e a relação dos conteúdos das representações sociais ${ }^{(8)}$. O núcleo central tem duas funções fundamentais: a geradora e a organizadora. A primeira trata da função geradora Que Qualifica, cria ou transforma o significado de todos os elementos constitutivos da representação em função das relações do objeto de RS com o sistema de valores e normas sociais. A segunda trata da função organizadora determinando a natureza dos elos, unindo e

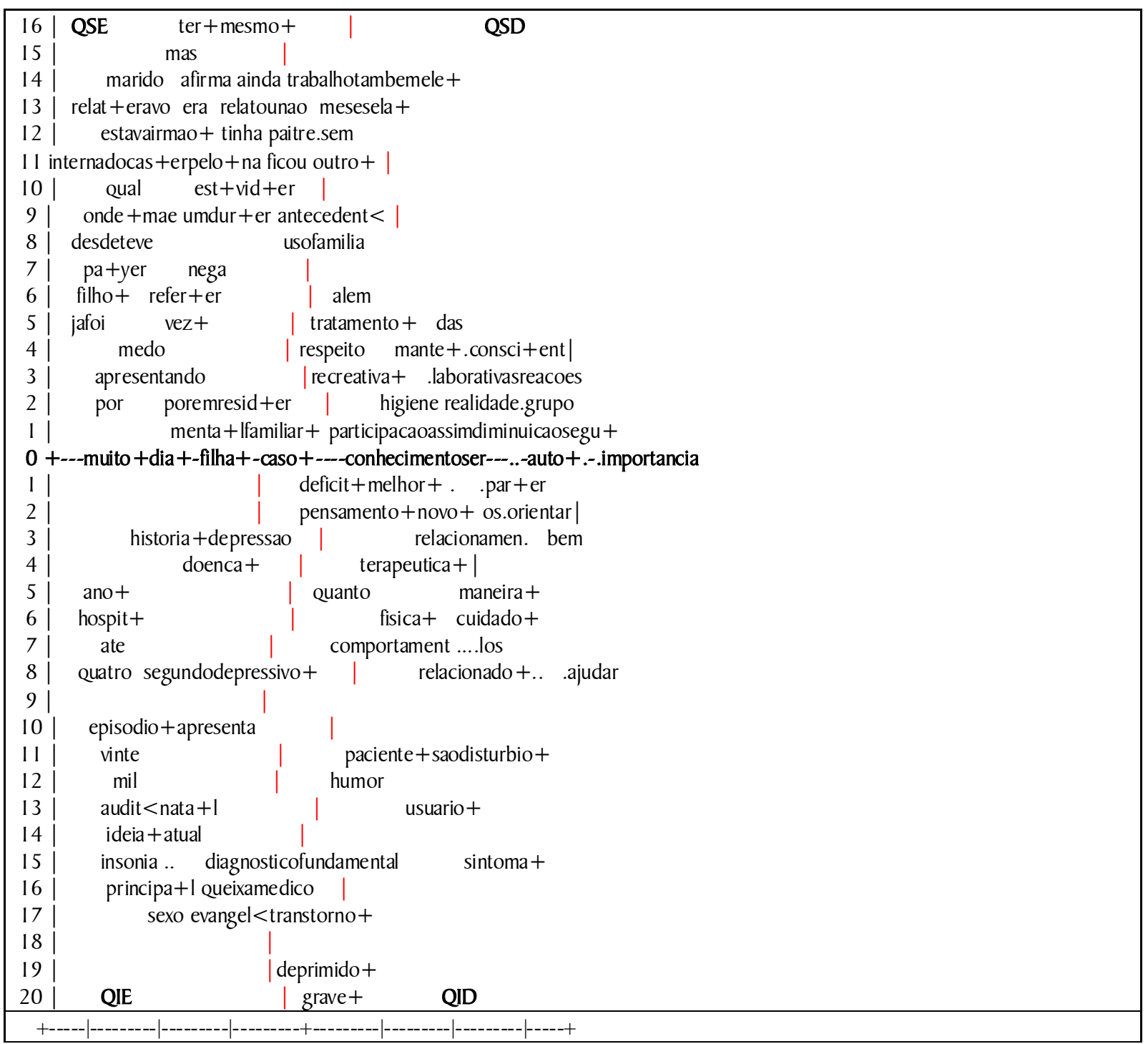

Figura 1. O papel terapêutico do agir do acadêmico de enfermagem da UFRN. 
estabilizando os diferentes elementos da representação.

Adicionamos na imagem gerada pelo ALCESTE (Figura I) alguns elementos gráficos, acrescentando-lhe uma abordagem particular dos autores Quando, ousadamente, faz uma analogia entre o núcleo central e os elementos periféricos como estes dois Quadrantes (QSE, QID) comparando-os a uma ampulheta. Ao graficar a dinâmica entre o explícito e o implícito modifica o esquema original do cone investido de Pichón-Rivière ${ }^{(11)}$ sobre o vínculo ${ }^{(12)}$.

Para nós, ao assenhorearmos desse critério também, comungamos de que o explícito ocupa a base do cone onde é observável, obviamente ocupando a maior superfície visível, enquanto Que o implícito localiza-se no vértice, como algo que não se vê ou Que está aparentemente visualizado, deslocado do contexto geral, embora configura uma certa centralidade entre os dois elementos representacionais de uma forma direta e entre estes, e os elementos intermediários. Essa compreensão permite associar a idéia da dupla face constitutiva da representação social. Esclarecemos que usamos linhas contínuas para fazer a analogia entre o explícito e implícito, por não ser objeto de estudo de atividades de grupos, como proposto originalmente.

Na perspectiva da Teoria do Núcleo Central, a centralidade nuclear se refere a família, emerge como elemento estruturante através das redes explicativas das relações de parentalidade e amizade configurando o espaço do domínio domiciliar. Neste, as constantes modificações dos componentes da ambiência familial, tornam-se vulneráveis, fragilizados diante da crise situacional decorrente do transtorno mental, alterando seus padrões de funcionamento afetando as oportunidades, acarretando um ônus afetivo, econômico e social. A família como estrutura e destacada no Quadrante superior esQuerdo (QSE) foi considerada como núcleo central das representações sociais para este grupo de acadêmicos.

\section{Núcleo Central - Família - a convivência, laços de amizade e adoecimento}

Toda representação social está organizada em torno de um núcleo central, Que determina, ao mesmo tempo, sua significação e organização interna ${ }^{(8)}$. É um subconjunto da representação, composto de um ou alguns elementos cuja ausência desestruturaria a representação ou Ihe daria uma significação completamente diferente.

Selecionamos a partir das raízes semânticas analisadas pelo QuiQuadrado as falas dos sujeitos investigados Que foram (re)agrupadas por sinônimos. Possibilita um novo texto, embora monossilábico constituído basicamente por substantivos, reflete e reforça a idéia central de similitudes na produção de sentidos enquanto teoria implícita. Exemplo: (pais(9); pai(12); filho(5), filhos(12); criança(4);:D- familiar(5), familiares(12); genitora(5); avo(6) irmãos(5); domiciliar(3), domicilio( 1); trabalho(7); amiga(3) colegas(4); amigos(4); marido(7)). Este rearranjo facilitou a subcategorização das falas localizadas em cada Quadrante da Figura I. Os exemplos a seguir, revelam aspectos da esfera da convivência familiar e dos laços amizades e do sentimentos e mudanças comportamentais sobre o adoecimento:

A mesma afirma Que durante a adolescência gostava de ficar isolada, era reservada. Queria uma pessoa perfeita Que nunca a machucasse, não existia, ela preferia não se envolver (l 1934$)$.
Durante as crises ela agredia fisicamente os filhos e apresentando fala apresada e incoerente. Os filhos estão sob a guarda do pai. Com o uso da medicação as crises minimizaram. Já apresentou três crises convulsivas e Quando ocorre a crise demora a voltar ao estado consciente" (223 19).

Vale ressaltar Que as funções geradora e organizativa das representações sociais não se destacam claramente. Sua visualização se faz de forma relacional, ou seja, no sentido de complementaridade dando sustentação e cristalização do objeto representacional. Assim, entendemos Que o caráter consensual das representações padece de algumas variáveis, ou viés Que a torna dinâmica enQuanto opinião, atitude de um dado grupo sobre um objeto em função da sua multiplicidade de apreensão por ser de natureza individual e social.

As inserções e situações sociais não são jamais completamente idênticas, os múltiplos processos Que intervêm nas tomadas de posição são também variados ${ }^{(13)}$. Essa dupla fonte de variação pode gerar uma multiplicidade aparente de tomadas de posição Que são, entretanto produzidas a partir de princípios organizadores comuns. Nesse sentido, é a representação Que determina o significado do comportamento e da interação, e não o inverso ${ }^{(8,14)}$.

\section{Elementos Periféricos - formas adaptativas}

$\mathrm{O}$ Quadrante inferior direito (QID) é o Quadrante que comporta os elementos periféricos de uma representação social. Os elementos periféricos são a interface entre o núcleo central (reificado) e a realidade dinâmica em constante transformação( ${ }^{(8)}$.

Os elementos periféricos têm três funções. A primeira, diz respeito à função concretizadora Que se relaciona diretamente com o contexto. Por seu caráter dependente, é responsável pela ancoragem da representação na realidade. A segunda tem a função de regulação, portanto, exerce papel essencial na adaptação da representação às evoluções do contexto, incluindo novas informações ou transformações do meio ambiente e abrigando as contradições acerca da representação. Finalmente, a terceira, com função de defesa funcionando como um "para-choQue", conferindo ao núcleo central maior resistência às mudanças.

São estruturas psicossociais Que dão condições suportivas para os membros da família com transtorno mental. Como as mudanças são marcantes, múltiplas e significativas para eles, os conteúdos destacados como elementos periféricos refletem essa movimentação Que os sujeitos frente ao adoecimento psíquico e seu funcionamento social intentam equilibrar o convívio familiar e da doença mental. Assim, lançam mão de estratégias, Quer religiosa, Quer psicoterapêutica Que vai desde o estabelecimento do diagnóstico, a sintomatologia as configurações sociais de enfrentamento, além de buscar causas ou antecedentes familiares para explicar o acontecimento elegendo, isoladamente ou concomitantemente, múltiplos artifícios de enfrentamento e sobrevivência.

Observamos a dimensão de alternância dos modos e maneiras de enfrentar o transtorno mental. Eles refletem o agir terapêutico do acadêmico de enfermagem através das etapas do processo de enfermagem com ênfase nas Queixas Que geram os problemas de enfermagem sobre o transtorno mental. Os elementos periféricos dizem respeito à religiosidade, a condição diagnóstica como rótulo social e segregacional, a sintomatologia como definidora do adoecer $\mathrm{e}$, as relações de parentalidade e amizade. 
A condição religiosa foi mencionada espontaneamente pelos entrevistados como uma forma de proteção sobre si, o adoecimento e a família, uma maneira explicativa ou causal para estar doente.

HISTORICO. AFF, 24 anos, sexo masculino, solteiro, nascido em Cuiabá, estudou ate a primeira serie do ensino fundamental, desempregado e evangelico. Tem como Queixa principal a impossibilidade de continuar os estudos em virtude de sua limitação intelectual (151 34).

A condição diagnóstica como rótulo social e a segregação. $\mathrm{O}$ sentido de estar doente e semi-internado não melhora a compreensão do Quadro Que é relembrado pelo número de internações, das crises e dos diagnósticos severos da doença mental. Reconhecem Que o tratamento do Hospital-Dia se diferencia da forma tradicional centrado modelo hospitalocêntrico, destacando o percurso do adoecimento mental, a convivência com outras doenças associadas e o processo de envelhecimento.

Em semi-internamento no HOSPITAL-DIA ha Quarenta dias. Portadora de depressão grave recorrente aproximadamente dez anos. Queixa-se de profunda angustia, tristeza e sentimento de vazio. Já esteve em tratamento no hospital anteriormente por três vezes, sendo este Quarto internamento nos últimos três anos (371 31).

Os aspectos explícitos da sintomatologia definem a compreensão do adoecer, constituindo a expressão verdadeira, angustiante e suplicante do adoecimento. São espectros representativos da enorme dificuldade e complexidade em transpor o limiar de superação das manifestações psicopatológicas.

Observamos pontos do conflito interno e externo, ambos subjacentes do Quão doído é o viver sob o peso da doença mental. Por não ser diagnosticada no plano do exame físico e seus artefatos diagnósticos, situando-se, particularmente, no campo da subjetividade, Quer do profissional de saúde, Quer do portador e da família carrega consigo sempre um aspecto representacional da realidade psicossocial envolvida.

O conjunto semântico reflete a forma representacional de lidar com a situação, por ser imprevista e multidinâmica remete a todos uma condição de riscos, expectativas e fragilidades, pois cada nova crise exige dos envolvidos, especialmente a família, um alargamento dos limites da situação como forma de superação e resolutividade, mesmo Que estes sintomas o (re) ingresse no circuito terapêutico dos transtornos mentais.

Apresentando distúrbios persecutórios, solilóquios, neologismos e comportamento inadequado. Usuário com três internamentos nesse hospital. Queixa principal. Apresenta delírios de perseguição, mania de grandeza, se diz muito inteligente, insônia e manifestações de agressividade (l 6 32).

Consideramos as relações de parentalidade e amizade, e, particularmente, as de gênero como uma zona implícita Que está abaixo ou subliminar ao explícito definida pelos elementos periféricos. A família é o local onde os integrantes sabem algo sobre coisas que todos sabem, mas que nenhum deles ousa dizer, pois são fontes de mal-entendidos, os Quais devem ser mantidos em segredo. Dessa forma, os segredos do grupo familiar de cada um dos integrantes são interditos.

Por intermédio da filha, foi admitido do HOSPITAL_DIA, apresentando ansiedade e cefaléia intensa como Queixa principal. o diagnostico medico detectado foi episodio depressivo grave com sintomas psicoticos. PROBLEMAS DE ENFERMAGEM: humor deprimido (268 32).

\section{Elementos intermediários - expectativas positivas do tratamento.}

O Quadrante superior direito (QSD) e inferior esquerdo (QIE) localizam-se os elementos intermediários das representações sociais. São conteúdos mais flexíveis Que interagem como os elementos periféricos e o núcleo central, em graus variados de pressão a inferência, engajamento e dispersão da informação.

Os mesmos dizem respeito ao tratamento, o desenvolvimento da agenda terapêutica proposta pelo Hospital-Dia, a aproximação teórica-prática da disciplina no desenvolvimento no cenário das práticas, além das expectativas de impacto positivo na resolução da crise e seus processos de superação pessoal e profissional.

Do ponto de vista do agir terapêutico do acadêmico de enfermagem as falas são indicativas do modo como foi resolvido o problema de enfermagem a partir das informações coletadas pelo instrumento usado no cenário de prática.

Há uma forte evidência da atuação do acadêmico no contexto do Hospital-Dia, no desenvolvimento cotidiano das habilidades e competências, assim como para o domicílio através da visita domiciliária Que possibilita ao aluno uma compreensão do ambiente familiar, na perspectiva, de uma atuação profissional pautado na estratégia saúde da família.

Apresenta-se orientada auto e alopsiQuicamente, comunicativa somente Quando estimulada e com idéias de suicídio todos os dias, participa das atividades laborativas e recreativas sem Queixas. Refere sentir a boca seca, sendo orientada a aumentar a ingesta de água (244 78).

Visita domiciliar com orientações e cuidados de enfermagem sobre a patologia. Reinserção familiar na proposta terapêutica com participação nas atividades recreativas. Estimular a higiene oral e corporal. Esclarecer sobre os riscos da drogadição. Observar sinais e sintomas de reações adversas (34 48).

Do ponto de vista da agenda terapêutica as ações são reforçadas continuamente frente às atividades laborais e receacionais, especialmente nos casos de maior comprometimento no funcionamento da socialização individual e grupal. À medida Que o tratamento avança na resolução da crise, o funcionamento do usuário melhora progressivamente, especialmente Quando associado às outras formas psicoterápicas e psicodinâmicas de tratar dos transtornos mentais.

Participa com entusiasmo das oficinas e das atividades laborativas da instituição como forma de inter-relacionamento e terapia ocupacional... encontra-se bem comunicativo e interagindo bem com os demais colegas e com a equipe (32 I 34). 
Participativo das atividades laborativas, todavia nas recreativas o mesmo não interage tanto (113 16).

Observamos uma atuação compreensiva do transtorno mental para além da pura técnica, mas na valorização dos aspectos afetivos, cognitivos e psicomotor, onde o relacionamento constitui uma técnica terapêutica eficaz na compreensão das manifestações psicopatológicas e do agir terapêutico no processo de cuidar nos casos dos transtornos mentais.

Estabelecer uma relação terapêutica baseada na confiança e no reconhecimento de suas Qualidades e capacidades, reforçandoas, personalizar o autocuidado e o valor do cliente (231 49).

Manter o paciente o mais independente pelo maior prazo de tempo possível, encorajando-o a fazer o máximo possível a cada dia (135 38).

O processo de cuidar em psieuiatria e na saúde mental está assentado na capacidade técnica do agir terapêutico frente às demandas da pessoa portadora dos transtornos mentais, pois no processo, no aQui-e-agora, na relação de ajuda os acontecimentos históricos de cada um dos integrantes são ritualizados e revividos através da comunicação e dos atos representacionais manifestos sobre o conhecimento ou desconhecimento do problema. Isto reflete a singularidade dos sujeitos psicossociais.

Nesse estudo, percebemos Que há uma produção de orientação e sentido para fortalecer as estruturas de superação das dificuldades enfrentadas pelo paciente e sua família, demonstrando uma preocupação na revalorização identitária enQuanto sujeito e membro de um grupo social definido. Portanto, a família constitui um grupo considerado primário. Nele os sujeitos têm papéis bastante definidos no dinamismo familiar desde a infância e Que mal-adaptados e não trabalhados no desenvolvimento e crescimento tornam-se fontes de estrangulamento emocional.

Frente às situações de mudança, surgem os medos básicos: o medo da perda e o medo do ataque ${ }^{(12)}$. O primeiro é o medo de perde o Que já se tem (marcos referenciais prévios, benefícios secundários do sintoma, adaptações passivas a situações de enfermidade, etc.). O segundo é o temor frente ao desconhecido, Que pode ser perigoso, e diante do Qual sentimos Que não estamos instrumentados para manejar com a nova situação.

Focalizar a responsabilidade para a mudança do cliente. Ajudar a identificar os meios de reestruturação do pensamento. Modificar o comportamento e usar os recursos. Encorajar a atividade física para descarregar a energia. Incluir outras pessoas significativas, como recursos e apoio social para ajudar a cliente a aprender novas respostas de adequação (383 66).

O cuidado físico e a terapias somáticas são necessárias para superar esses problemas. Essas estratégias ajudam a aumentar a sensação de controle do cliente sobre os objetivos $e$ comportamentos, estimular a autoestima e modificar as expectativas negativas (236 47).

Estimular a participação do paciente nas atividades, oficinas e grupo, havendo maior interação entre eles. Trabalhar em parceria com terapia ocupacional, psicologia e psiQuiatria (355 37).

O agir terapêutico do acadêmico de enfermagem, por ser uma prática em construção, situou-se nos Quadrantes definidores dos elementos intermediários. Dada sua fluidez na composição das representações sociais e das práticas de enfermagem no cuidado aos transtornos mentais, salientamos Que não poderia ser de outra forma, pois não há solidez na conformação do núcleo central, embora, mantenha o movimento psicodinâmico através do grau de inferência e pressão sobre o núcleo. Os elementos intermediários como algo novo revela a dupla face da representação Que enfoca o agir terapêutico na saúde mental no aspecto figurativo e produtor de sentido.

Dessa forma, a existência deste duplo sistema permite compreender Que as representações sejam, simultaneamente, estáveis e móveis, rígidas e flexíveis ${ }^{(8)}$. Entendemos Que as representações sociais e as práticas do agir terapêutico do acadêmico de enfermagem recebem influências de esQuemas figurativos de outros modos, também, multifacetados da própria representação arQuetípica da doença mental.

\section{CONSIDERAÇÕES FINAIS}

\section{$\mathbf{O} \underline{X}$ da Questão Representacional}

Analisamos as representações na perspectiva da Teoria do Núcleo Central mediante os Quadrantes gerados pelo ALCESTE destacase um termo justaposto, "conhecimentoser". A dupla face revela Que as representações sociais e as práticas disciplinares em Saúde Mental com ênfase no papel terapêutico dos acadêmicos de enfermagem requerem conhecimento para ser. Ao reportarmos a Figura I indica Que modificamos a figura para olhar o núcleo central, os elementos periféricos e os elementos intermediários, traçando uma linha imaginária.

Salientamos Que ao entrecruzar os Quadrantes e seus respectivos conteúdos formando um $\underline{\mathrm{X}}$ a informação central remete ao entendimento representacional para este grupo de acadêmicos. Nesse sentido, os elementos intermediários podem ser a porção mais implícita da representação social, (re)atualizando com sucessivas aproximações e afastamentos aQuilo Que é considerado explícito, o transtorno mental e toda sua complexidade, polissemia e ambivalência.

Inferimos Que para este grupo de alunos investigados a partir da análise das atividades práticas realizadas no cenário do HospitalDia a representação social é estabelecida entre adQuirir conhecimento para ser terapêutico, como aluno e como profissional na medida em Que a família circunscreve o objeto do cuidar em enfermagem ao adotar formas adaptativas e das expectativas positivas do tratamento. Esta competência o torna hábil para lidar com os transtornos mentais na perspectiva deste serviço intermediário, assim como no modelo oficial de atenção à saúde com compromisso e responsabilidade social no exercício político das ações promocionais de atenção a saúde e o agir terapêutico mediante a responsabilização, o vínculo e o contrato enQuanto critérios norteadores da integralidade das ações. Estas, requeridas pelo processo saúde-doença.

Entendemos Que os achados desse estudo, mesmo insipientes, referenda a metodologia adotada na área de Saúde Mental no 
contexto da Clínica Ampliada, bem como das constantes adeQuações e inovações operacionais introduzidas no Projeto Político Pedagógico do Curso de Graduação em Enfermagem da UFRN. Assim, como uma estratégia nos serviços de saúde nos Quais (co)existem aproximações operacionais, teóricas e metodológicas frente aos princípios e diretrizes do Sistema Único de Saúde.

\section{REFERÊNCIAS}

1. Ministério da Saúde (BR). Reorganização do sistema de saúde mental: uma experiência do Qualis2. Rev Promoção Saúde 2000; 2(3): 446.

2. Ministério da Saúde (BR). Secretaria Executiva. Núcleo Técnico da Política Nacional de Humanização. HumanizaSuS: acolhimento com avaliação e classificação de risco: um paradigma ético-estético no fazer em saúde. Ministério da Saúde, Secretaria-Executiva, Núcleo Técnico da Política Nacional de Humanização. Brasília: Ministério da Saúde; 2004.

3. Scóz TMX, Fenili RM. Como desenvolver projetos de atenção à saúde mental no programa de saúde da família. Rev Eletrôn Enferm 2003; 5(2): 7I-7.

4. Jodelet D. Folie et représentations sociales. Paris: PUF; 1989.

5. Moscovici S. A representação social da psicanálise. Rio de Janeiro: Zahar; 1978.

6. Arruda A. Representando a alteridade. Petrópolis: Vozes; 1998.

7. Wagner W, Elejabarrieta F. Representações Sociais. In: Morales IP. Psicologia Social. Madrid: McGraw-Hill; 1994.

8. Abric JC. Lês représentations sociales: aspects théoriQues. In:
Abric IC. Pratieues sociales et représentations. Paris: Press Universitaires de France; 1994.

9. Miranda FAN. Representações sociais sobre a atuação do enfermeiro psiQuiátrico no cotidiano [tese]. Ribeirão Preto (SP). Escola de Enfermagem de Ribeirão Preto, Universidade de São Paulo; 2002.

10. Camargo BV. ALCESTE: um programa informático de análise Quantitativa de dados textuais. Florianópolis: Universidade Federal de Santa Catarina; 1998.

11. Pichón-Rivière E. La noción de tarea en psieuiatía - el proceso grupal. Buenos Ayres: Nova Vision; 1977.

12. Berstein M. Contribuições de Pichón-Rivière à psicoterapia de grupo. In: Osório LC. Grupoterapia hoje. Porto Alegre: Artes Médicas; 1989.

13. Doise W. Lês représentations sociales. In: Ge R, Bonnet C, Richard JF. Traité de psychologie cognitive. Paris: Dunod; 1990. p.157-70.

14. Sá CP. A Construção do objeto de pesQuisa em representações sociais. Rio de Janeiro: Eduerj; 1998. 
